\title{
Heat transfer enhancement in dense suspensions of agitated solids. Part I: Theory.
}

\author{
Xinglong Chen $^{\mathrm{a}}$ and Michel Louge ${ }^{\mathrm{a} *}$

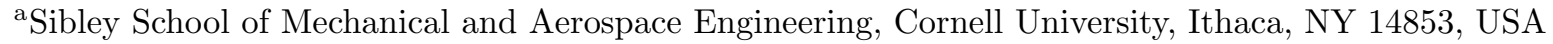

\begin{abstract}
We predict heat transfer enhancement through dense homogeneous suspensions of agitated solids in conductive fluids by coupling the fluid and solid phases through a volumetric source term. The enhancement is governed by a Damköhler number demarcating an "exchange limit" where the source term dominates, and a "diffusion limit" set by the ability of agitated particles to self-diffuse. We point out effects of particle ordering on mixture conductivity and volumetric heat exchange rate, carry out thermal simulations to justify the form of these terms, and model further enhancements from gas velocity fluctuations induced by solids of high agitation.
\end{abstract}

keywords: fluid-solid heat transfer, granular agitation, exchange limit, diffusion limit

\section{Introduction}

Heat transfer in fluid flows heavily laden with solid particles is crucial to processes in the chemical, mining, power, pharmaceutical, food and oil industries, as well as in the combustion of solid fuels. Accordingly, heat transfer in gas-solid mixtures has been the object of several experiments, see for example Glicksman [1] and Molerus and Wirth in fluidized beds [2], Jepson, Poll and Smith [3] in pneumatic transport, and Patton, Sabersky and Brennen [4] and Natarajan and Hunt [5] in granular flows. Numerical simulations also began to address how idealized particles exchange heat with a wall and the surrounding fluid [6].

In Part I of this paper, we consider homogeneous dense suspensions of agitated particles in a gas. For simplicity, we restrict attention to systems in which there is no difference between the average velocity of the solids and gas. By agitation we mean that particles have significant fluctuation velocities about the average. For nearly spherical particles with relatively large Stokes number [7-9], these fluctuations arise from inter-particle collisions. This has led to theories in which the particle phase is modeled with ele-

${ }^{*}$ Corresponding author. Voice (607) 255 4193; fax (607) 255 1222; electronic mail: Michel.Louge@cornell.edu. ments of the kinetic theory, while the flow is assumed to be laminar [10] or turbulent [11-15]. In such flows, particle agitation is measured with the "granular temperature" $\Theta \equiv(1 / 3) \overline{v_{i}^{\prime} v_{i}^{\prime}}$, where $v_{i}^{\prime}$ is the particle fluctuation velocity in the cartesian direction $i$. It is with this parameter that the solid phase can transmit momentum through an effective viscosity. The granular temperature owes its name to an analogy with the translational temperature that is defined in the kinetic theory for a gas of hard spheres. Thus, $\Theta$ bears no relation to the usual thermal temperature of the solids, which we will later denote with the distinct symbol $T_{s}$.

Collisions also give rise to an effective conductivity of the solid phase that enhances the net transfer of thermal energy [16]. However, as Sun and Chen showed [17], individual impacts are too short in most cases to permit the conduction of any significant heat between particles during their ephemeral contact. Instead, the collisional enhancement of the thermal heat flux first involves an exchange of heat with the gas, and then the gas transfers its energy to the wall. Accordingly, Louge, Mohd. Yusof and Jenkins developed a theory that identifies distinct thermal temperatures for the gas and solids phases [18]. Its mechanism is twofold.

First, the granular agitation induces the self- 
Table 1

Nomenclature

$a_{0}$

$a_{\nu}, b_{\nu}, c_{\nu}, d_{\nu}$

$A, A_{s}$

$A_{1}, A_{2}, A_{3}$

$c_{g}, c_{s}$

$d$

$D_{s}$

e

$E_{1}, E_{2}, E^{*}$

$f, f_{c}$

$g_{0}, g_{12}$

$h$

$h_{11}, g_{11}$

H

$i, j, k$

$I_{a}, I_{\nu}$

$k_{g}, k_{s}$

$k_{\text {eff }}$

$k_{t}, k_{g t}$

$K_{g}, K_{s}$

$K_{g t}$

$\ell^{*}$

$L$

$L^{\dagger}$

$L_{0}$

$M$

$n$

$N$

$q_{0}, q$

$q_{D}, q_{E}$

$\dot{q}_{s}$

$Q_{0}, Q$

$r$

$R_{\text {diss }}$

$S$

$t$

$T_{g}, T_{s}$

$T^{+}, T^{-}$

$v_{i}^{\prime}, u_{i}^{\prime}$

$V_{s}$

$w^{*}$

$x_{i}$

$x, y, z$

$y_{c}$ vibration amplitude

constants in Eq. (48)

grain surface areas

functions in Eq. (4)

fluid, solid specific heats per mass

grain diameter

granular self-diffusivity

normal restitution coefficient

stiffnesses

vibration, impact frequencies

wall, binary pair distributions

heat transfer coefficient

functions in Eq. (27)

volumetric rate of heat exchange

indices

integrals in Eqs. (29), (31)

gas, solid thermal conductivities

effective thermal conductivity

turbulent, total fluid conductivities

mixture, solid-phase conductivities

augmented mixture conductivity

dimensionless decay length of $\Theta$

channel width

dimensionless length scale in Eq. (16)

reference width in Eq. (48)

constant in $\kappa$ from Eq. (58)

particle number density

number of spheres

clear gas, suspension wall heat fluxes

wall flux in diffusion, exchange limits

heat rate

cumulative heat exchanged

radial coordinate

dissipation function $[7,8]$

wall, strip areas

time

gas, solid thermal temperatures

temperatures at $y= \pm L / 2$

particle, fluid fluctuation velocities

sphere volume within a strip

$\sqrt{\Theta / \Theta_{0}}$

$i$-coordinate

cartesian coordinates

sphere center coordinate
Table 2

Greek

$\alpha_{s} \quad$ granular thermal diffusivity

$\alpha_{1} \quad$ function in Eq. (27)

$\gamma_{\text {vis }}, \gamma$ granular energy viscous, collisional dissipation rates

$\Gamma_{u} \quad$ strain rate

$\epsilon_{m} \quad$ non-continuum lubrication parameter

$\zeta \quad$ relative distance to first center (Eq. 25)

$\theta_{1}, \theta_{2} \quad$ functions in Eq. (27)

$\Theta, \Theta_{0}$ granular temperatures

$\kappa \quad$ conductivity of granular fluctuation energy

$\lambda_{s}, \lambda_{g}$ grain, gas mean free paths

$\mu \quad$ fluid viscosity

$\mu_{t} \quad$ eddy viscosity

$\nu \quad$ solid volume fraction

$\xi_{s} \quad k_{s} / k_{g}$

$\xi_{s t} \quad k_{s} / k_{g t}$

$\xi_{i} \quad$ roots of $\xi / \tan \xi=1-\mathrm{Bi}$

$\rho_{g}, \rho_{s} \quad$ fluid, solid material densities

$\sigma_{1}, \sigma_{2} \quad$ Poisson's ratios

$\tau \quad$ flow characteristic time

$\tau_{c}, \tau_{e} \quad$ collision, contact conduction times

$\omega \quad$ constant in Eq. (55)

Table 3

Dimensionless groups

$\mathrm{Bi} \quad$ Biot number

Da Damköhler second ratio

Fo, $\mathrm{Fo}_{c} \quad$ Fourier numbers

Kn Knudsen number

$\mathrm{Le}_{s} \quad$ granular Lewis number

$\mathrm{Nu}, \mathrm{Nu}_{L} \quad$ particle, channel Nusselt numbers

$\mathrm{Nu}_{\text {eff }} \quad$ effective particle Nusselt number

$\mathrm{Pr} \quad$ Prandtl number

$\mathrm{Pr}_{t} \quad$ turbulent Prandtl number

Re particle Reynolds number

St, $\mathrm{St}_{\gamma} \quad$ Stokes numbers

Table 4

Scripts

$\dagger, *$

$\sim$

g, $\mathrm{s}$ dimensionless oscillating quantity gas, solid 
diffusion of solid particles [19] across regions with different thermal temperatures. This transport is not driven by a gradient of particle concentration, but it is responsible for the effective conductivity of the agitated solid phase. If the latter possesses a gradient of thermal energy, particle selfdiffusion can drive a heat flux through it, even if the solid concentration is uniform. Second, because particles have a finite thermal inertia, they do not adopt instantly the temperature of the surrounding gas. The resulting temperature difference then drives a thermal exchange between gas and solids.

Near the wall, this exchange amplifies the gas temperature gradient, as particles from warmer regions, for example, bring energy closer to a cold wall than what the conductivity of the gas would alone accomplish. Thus, although agitated solids do not themselves exchange any heat with the wall during their direct, ephemeral contact with it, their self-diffusion from other regions can raise the heat transfer through the gas.

Therefore, in this view, the overall heat transfer at the wall depends on the energy exchanged between gas and solids with distinct thermal temperatures, as well as on the magnitude of the selfdiffusion of the agitated grains. It is the competition between these two rate-limiting processes near the wall that determines the regime of heat transfer enhancement that is observed there.

Another approach is to regard the suspension as a medium endowed with a single thermal temperature and an enhanced mixture thermal conductivity [20]. While useful in the bulk, that approach requires a regularization of the thermal boundary condition at a wall to account for the role of particle agitation [21]. As we will show, it is only appropriate in the limit where diffusion dominates.

In Part I of this paper, we first examine the competition between thermal exchange and particle self-diffusion with a generic analysis of agitated spherical grains suspended in a fluid. By analogy with the second Damköhler ratio, we derive a number gauging the relative importance of the two mechanisms.

Using relatively crude numerical simulations, we then test the origin of the thermal exchange rate and the role of the Biot and Fourier numbers for individual grains. Near the flat wall, we show that the exchange is affected by the pair distribution at contact, which induces spatial fluctuations of available surface area and volume. We model the role of the conductive grains in enhancing the static mixture conductivity in the continuous phase. Finally, we identify phenomena that complicate our generic analysis at high solids agitation by augmenting the effective gas conductivity in the wake of moving grains.

In Part II, we will describe an experiment designed to test the theory with a vibrated box heavily laden with macroscopic spherical grains. Because energetic vibrations are required to maintain the grains agitated in the presence of gravity, particle self-diffusion is so intense that the experiment resides in the "exchange limit", where heat transfer enhancement is set by the volumetric rate of energy exchanged between gas and solids. The high granular agitation also augments the mixture conductivity and the rate of heat transfer between individual grains and the gas by raising particle-induced gas velocity fluctuations.

We will focus on the "diffusion limit" in Part III. There are roughly two ways to reach this limit. The first, which employs macroscopic grains, would be to carry out experiments in microgravity, so that agitation could be reduced without collapsing the suspension. The second is to shrink the system size. In Part III, we will illustrate the latter by considering colloidal suspensions of nanoparticles.

We begin Part I by illustrating the exchange and diffusion limits with a simple analysis of the heat transfer enhancement at a wall confining a fluid laden with agitated particles.

\section{Generic model of thermal enhancement}

Consider a uniform suspension of grains of diameter $d$, material density $\rho_{s}$, material conductivity $k_{s}$ and material specific heat $c_{s}$ agitated at a constant "granular temperature" $\Theta$ in a fluid of thermal conductivity $k_{g}$ between two infinite parallel flat walls separated by a distance $L$. We set the origin midway between the walls located 
at $y= \pm L / 2$. Variables are only function of the thermal gradient direction $y$, the phenomenon is steady on time scales $\gg d / \sqrt{\Theta}$, and there is no mean relative velocity between grains and fluid. On planes of constant $y$, the average thermal temperature of the fluid is $T_{g}$ and that of the grains is $T_{s}$. The uniform solid volume fraction is $\nu$ and the number density of grains is $n=6 \nu / \pi d^{3}$.

In this simple picture, the thermal energy ODEs for the fluid and granular phases are, respectively,

$$
0=-\frac{d}{d y}\left(-K_{g} \frac{d T_{g}}{d y}\right)+H,
$$

and

$$
0=-\frac{d}{d y}\left(-K_{s} \frac{d T_{s}}{d y}\right)-H,
$$

where $H$ is the average volumetric rate of thermal energy given by the grains to the fluid, $K_{g}$ is the conductivity of the gas-solid mixture, and $K_{s}$ is the effective conductivity of the granular phase. We will revisit these thermal governing equations in Part III, for cases in which $\nu$ is no longer uniform.

Grains of Biot number $\mathrm{Bi} \equiv h(d / 2) / k_{s} \ll 1$ have nearly uniform internal temperature $T_{s}$. By exchanging heat with their surroundings through the surface of area $A=\pi d^{2}$ at a convection coefficient $h$, they contribute, on average, to a volumetric rate $[5,18,20,21]$

$$
H=n A h\left(T_{s}-T_{g}\right)=12 \nu \frac{k_{g}}{d^{2}} \mathrm{Nu}\left(T_{s}-T_{g}\right),
$$

where, for now, we take the grain surface area $n A$ available for heat exchange with the fluid in a unit volume to be invariant, and the Nusselt number $\mathrm{Nu} \equiv h(d / 2) / k_{g}$ to be unity and to be based on the molecular conductivity $k_{g}$ of the gas. We will re-examine these assumptions later.

We distinguish the mixture conductivity $K_{g}$ of the fluid phase from the material conductivity $k_{g}$ of the pure fluid. While $k_{g}$ is invariant, $K_{g}$ is affected by the presence of particles. At negligible agitation, it is well approximated by homogenization models inspired by that of Maxwell [22]. For spherical grains, we adopt the semi-empirical expression of Meredith and Tobias [23] for $K_{g}$, which is equivalent to Maxwell's original model at low $\nu$ and $\xi_{s} \equiv k_{s} / k_{g}$, but performs better at $\nu>0.1$ and $\xi_{s}>10$ [24],

$$
\frac{K_{g}}{k_{g}}=\frac{A_{1}-2 \nu+A_{2}-2.133 A_{3}}{A_{1}+\nu+A_{2}-0.906 A_{3}} \equiv f_{M}\left(\nu ; \xi_{s}\right),
$$

where $A_{1}=\left(2+\xi_{s}\right) /\left(1-\xi_{s}\right), A_{2}=0.409 \nu^{7 / 3}(6+$ $\left.3 \xi_{s}\right) /\left(4+3 \xi_{s}\right)$, and $A_{3}=3 \nu^{10 / 3}\left(1-\xi_{s}\right) /\left(4+3 \xi_{s}\right)$. At high agitation, $K_{g}$ is also influenced by gas velocity fluctuations induced by the moving grains.

Granular agitation gives rise to self-diffusion of the grains with coefficient $[18,19]$

$$
D_{s}=\frac{d \sqrt{\Theta}}{(9 \sqrt{\pi}) \nu g_{12}}\left(\frac{1}{1+2 \mathrm{Kn}}\right),
$$

where $g_{12}(\nu)$ is the pair distribution of colliding spheres "1" and "2" at contact, which, for two identical spheres, is well represented by the Carnahan and Starling expression [25]

$$
g_{12}=\frac{2-\nu}{2(1-\nu)^{3}},
$$

as long as $\nu$ stays below the "freezing" value $\approx$ $49 \%$ [26]. In Eq. (5), the term in parentheses is a correction for high Knudsen number $\mathrm{Kn}=$ $\lambda_{s} / L$ that is significant when the granular mean free path $\lambda_{s}=d /\left[6 \sqrt{2} \nu g_{12}\right]$ between consecutive impacts is on the order of the vessel size $L[18,27]$. The self-diffusion leads to a thermal diffusivity of the dispersed solid phase [18],

$$
\alpha_{s} \equiv \frac{K_{s}}{\rho_{s} \nu c_{s}}=D_{s} / \mathrm{Le}_{s}
$$

where $\mathrm{Le}_{s} \equiv D_{s} \rho_{s} \nu c_{s} / K_{s}$ is a granular Lewis number that we take equal to one.

The crucial assumption is that grains collide too quickly to exchange any significant thermal energy with each other or with the wall $[17,18]$. As these references indicate, it is valid as long as the time of particle contact in an impact

$$
\tau_{c} \sim \frac{\rho_{s}^{2 / 5} d}{\Theta^{1 / 10} E^{* 2 / 5}}
$$

is much smaller than the time to equilibrate the particle temperature by conduction through the 
area of contact

$$
\tau_{e} \sim \frac{\rho_{s}^{3 / 5} E^{* 2 / 5} d^{2} c_{s}}{\Theta^{2 / 5} k_{s}}
$$

where $E^{*-1} \equiv\left[\left(1-\sigma_{1}^{2}\right) / E_{1}+\left(1-\sigma_{2}^{2}\right) / E_{2}\right]$ is a reduced inverse stiffness that combines the Young's moduli $E_{i}$ and Poisson's ratios $\sigma_{i}$ for the two impact protagonists of indices 1 and 2. Thus, our assumption is valid as long as $\tau_{c} \ll \tau_{e}$ or, equivalently, when

$$
\frac{\rho_{s}^{1 / 5} E^{* 4 / 5} d c_{s}}{\Theta^{3 / 10} k_{s}} \gg 1
$$

For spheres engaged in violent, non-Hertzian impacts, the experiments of Ben-Ammar, et al [28] indicate how plastic yield can decrease $\tau_{e}$, thus making it more difficult to uphold condition (10).

We solve Eqs. (1)-(3) subject to the following boundary conditions at the two confining walls: prescribed fluid temperatures,

$$
T_{g}(y= \pm L / 2)=T^{ \pm}
$$

and vanishing fluxes of thermal energy through the solid phase or, with $K_{s} \neq 0$,

$$
\frac{d T_{s}}{d y}(y= \pm L / 2)=0,
$$

which reflect the lack of direct thermal energy transfer in ephemeral collisions between the grains and the wall. Defining the dimensionless thermal temperatures of the fluid and solid phases

$$
T_{g, s}^{\dagger} \equiv \frac{T_{g, s}-\left(T^{+}+T^{-}\right) / 2}{\left(T^{+}-T^{-}\right)},
$$

and the dimensionless distance $y^{\dagger} \equiv y / L$, we write the solution as

$$
T_{s}^{\dagger}=\frac{y^{\dagger} L^{\dagger} \cosh \left(L^{\dagger} / 2\right)-\sinh \left(y^{\dagger} L^{\dagger}\right)}{L^{\dagger} \cosh \left(L^{\dagger} / 2\right)+2\left(K_{s} / K_{g}\right) \sinh \left(L^{\dagger} / 2\right)},
$$

and

$$
T_{g}^{\dagger}=\frac{y^{\dagger} L^{\dagger} \cosh \left(L^{\dagger} / 2\right)+\left(K_{s} / K_{g}\right) \sinh \left(y^{\dagger} L^{\dagger}\right)}{L^{\dagger} \cosh \left(L^{\dagger} / 2\right)+2\left(K_{s} / K_{g}\right) \sinh \left(L^{\dagger} / 2\right)},
$$

where the dimensionless scale of the system is

$$
L^{\dagger} \equiv \frac{L}{d} \sqrt{12 \nu \mathrm{Nu}\left(\frac{k_{g}}{K_{g}}+\frac{k_{g}}{K_{s}}\right)} .
$$

In the absence of particles, the temperature would vary linearly between $y= \pm L / 2$. Therefore, with a pure fluid, the heat flux at either wall would be

$$
q_{0}=-\left.k_{g} \frac{d T_{g}}{d y}\right|_{ \pm y / 2}=-k_{g} \frac{\left(T^{+}-T^{-}\right)}{L} .
$$

Our assumption is that particles do not contribute to the heat flux $q$ through the wall during their ephemeral contacts with the latter. However, they affect the mixture conductivity. Thus, with particles, the wall flux is

$$
q=-\left.K_{g} \frac{d T_{g}}{d y}\right|_{ \pm y / 2}
$$

Then, differentiating Eq. (15), the thermal enhancement can be written as the ratio

$$
\frac{q}{q_{0}}=\left(\frac{K_{g}}{k_{g}}\right)\left(\frac{1+\frac{K_{s}}{K_{g}}}{1+\frac{K_{s}}{K_{g}} \frac{\tanh \left(L^{\dagger} / 2\right)}{\left(L^{\dagger} / 2\right)}}\right) .
$$

This expression may be interpreted as a dimensionless effective conductivity of the suspension, or as an overall Nusselt number based on the channel's half-width, $\mathrm{Nu}_{L} \equiv k_{\text {eff }} / k_{g} \equiv q / q_{0}$. Because the mixture is at rest, $T_{s}$ and $T_{g}$ vary only along $y$, and, unlike laminar convection in a pipe, $\mathrm{Nu}_{L}$ remains the same whether a constant flux, or a constant temperature, is imposed at the wall. The first term in Eq. (19), $K_{g} / k_{g}$, represents changes in the mixture fluid conductivity due to the mere presence of particles. This can arise from conduction through the grain material, as captured by static homogenization models (section 3.1), or from changes in the fluid conductivity associated with, for example, fluid velocity fluctuations induced by grain agitation (section 3.4 ).

The second term in parentheses represents the additional enhancement due to granular thermal self-diffusion. Because grains do not transfer any 
appreciable heat directly to the wall, their selfdiffusion can only increase the overall heat transfer, once they have exchanged heat with the surrounding fluid. By analogy with diffusion flames, where either chemical kinetics or diffusion can be rate-limiting, we define a Damköhler second ratio

$$
\mathrm{Da} \equiv \frac{\left(K_{g} / K_{s}\right)\left(L^{\dagger} / 2\right)}{\tanh \left(L^{\dagger} / 2\right)} .
$$

Values of $\mathrm{Da} \rightarrow 0$ occur in flows with considerable granular agitation $\left(K_{s} \gg K_{g}\right)$. In this limit, $L^{\dagger}$ and $q / q_{0}$ become independent of $K_{s}$,

$$
\frac{q_{E}}{q_{0}} \equiv \lim _{K_{s} / K_{g} \rightarrow \infty}\left(\frac{q}{q_{0}}\right)=\left(\frac{K_{g}}{k_{g}}\right) \frac{\left(L^{\dagger} / 2\right)}{\tanh \left(L^{\dagger} / 2\right)},
$$

so that agitation is not rate-limiting. Instead, for a given $K_{g}$ and in a vessel of finite size, because

$$
\frac{\partial\left(q_{E} / q_{0}\right)}{\left(q_{E} / q_{0}\right)}=\left(\frac{\partial L^{\dagger}}{L^{\dagger}}\right)\left(1-\frac{L^{\dagger}}{\sinh L^{\dagger}}\right) \approx \frac{\partial L^{\dagger}}{L^{\dagger}}=\frac{1}{2} \frac{\partial H}{H},
$$

the ratio $q_{E} / q_{0}$ is governed by the grains' ability to exchange heat with the surrounding fluid through $H$ in Eqs. (1) and (2). We call this regime the "exchange limit."

In contrast, values of Da $\gg 1$ occur in systems where the vessel size greatly exceeds the particle diameter $\left(L^{\dagger} \gg 1\right)$. Here, $q / q_{0}$ becomes independent of $L^{\dagger}$ or $H$,

$$
\frac{q_{D}}{q_{0}} \equiv \lim _{L^{\dagger} \rightarrow \infty}\left(\frac{q}{q_{0}}\right)=\left(\frac{K_{g}}{k_{g}}+\frac{K_{s}}{k_{g}}\right),
$$

and it is no longer necessary to distinguish the thermal temperatures of the fluid and solid phases, $T_{g} \simeq T_{s}$. In this regime, which we call the "diffusion limit," the heat flux ratio increases with the conductivity $K_{g}$ of the fluid-solid mixture augmented, if particles are sufficiently agitated, by the self-diffusive conductivity $K_{s}$. If they are not,

$$
\lim _{K_{s} \rightarrow 0}\left(\frac{q_{D}}{q_{0}}\right)=\left(\frac{K_{g}}{k_{g}}\right) .
$$

As we will discuss in Part III, equations (23) or (24) are relevant to suspensions of nanoparticles.
The diffusion limit, for which $K_{s}$ matters but not $H$, should also be accessible in microgravity suspensions of macroscopic grains, where relatively modest agitation can be generated without collapsing the suspension.

Figure 1 illustrates the transition between the two limits and the corresponding dimensionless profiles of fluid and grain thermal temperatures. In the diffusion limit with large Da, profiles of fluid and solid thermal temperatures are identical except near the walls (Fig. 1, top left). As Da decreases, the distinction becomes more pronounced (Fig. 1, top middle). In the exchange limit with small Da, solids have considerable selfdiffusive conductivity $K_{s}$, and thus exhibit nearly uniform temperature between hot and cold walls (Fig. 1, top right). By exchanging heat with the fluid, solids in the exchange limit steepen the fluid temperature gradient at the wall, and thus raise the wall heat transfer. For given $\nu$ and $L / d$, further steepening can occur if $K_{g}$ or $k_{g}$ are augmented by particle-induced fluid velocity fluctuations (section 3.4).

\section{Complications}

The picture presented in the previous section is complicated by local ordering induced by the flat thermal walls, and by the possible creation of fluid velocity fluctuations by fast-moving particles. Such fluctuations affect $H$ by raising the heat transfer coefficient around individual spheres. The ordering modifies the mixture conductivity $K_{g}$ and the source term $H$ by altering the available particle surface and volume. It is captured by theories for the pair distribution of hard spheres interacting with the wall. We consider the role of such ordering first. To avoid further complications, we ignore a particle size distribution, which would lead to segregation [9].

\subsection{Available surface and volume}

Because $H \propto n A$, the source term in Eqs. (1) and (2) depends on the external surface area that each grain has available to exchange heat with the surrounding fluid. Similarly, the homogenized expression for $K_{g}$ varies with local solid volume fraction. The ordering of spherical grains induced 


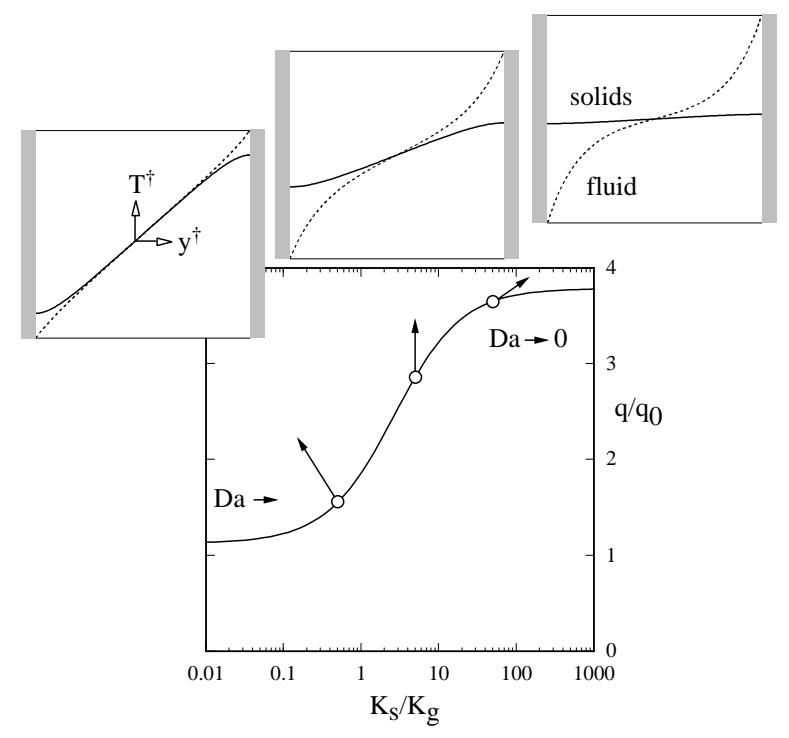

Figure 1. Dimensionless profiles of fluid and solids temperature, respectively $T_{g}^{\dagger}$ (dashed lines) and $T_{s}^{\dagger}$ (solid lines), along $y^{\dagger}$ (top three graphs), and flux ratio $q / q_{0}$ versus $K_{s} / K_{g}$ (bottom graph) for $K_{g} / k_{g}=1.13$ and $L / d=8$, corresponding to spheres of $3.18 \mathrm{~mm}$ uniformly agitated in a box with distance from hot to cold walls of $25.4 \mathrm{~mm}$ containing air and solids $\left(k_{s} / k_{g} \approx 6\right)$ at a volume fraction $\nu=6.5 \%$, like experiments described in Part II. We ignore for now the augmentation of $K_{g}$ or $k_{g}$ by solids agitation, and take $\mathrm{Nu}=1$. Circles on the bottom curve indicate the three distinct values of $K_{s} / K_{g}$ for the top profiles. The corresponding Damköhler numbers are, from left to right, $\mathrm{Da}=12,0.7$ and 0.07 . by our flat thermal walls provoke local oscillations of the probability to find particle centers within a given distance from such walls. In turn, these spatial variations let the grain surface area per unit volume $\widetilde{n A}$ and the fraction of the volume occupied by solids, which appears in Eq. (4), oscillate as well.

The oscillations, which we denote by a tilde, are captured by theories pioneered by Percus and Yevick (PY) [29,30] for hard spheres. For simplicity, we assume that the "bulk" solid volume fraction $\nu$ is invariant near the wall, and that any quantity varies only with $y$, the coordinate normal to and, in this case, originating at the wall. Because hard spheres cannot penetrate the latter, their centers lie at $y>d / 2$, and it is convenient to define the dimensionless distance $\zeta$ to the center of spheres touching the wall,

$$
\zeta \equiv \frac{y}{d}-\frac{1}{2} .
$$

The PY theory calculates the elementary number of spheres $d N$ with centers in the range $\zeta \in[\zeta, \zeta+$ $d \zeta$ ] per unit area $S$ of the wall as

$$
\frac{d N}{S}=\frac{6}{\pi d^{2}} \nu g_{0}(\zeta ; \nu) d \zeta,
$$

where $g_{0}$ is the spatial distribution of hard spheres interacting with the flat wall. Lack of penetration implies $g_{0}=0$ for $\zeta<0$.

The theory of Henderson, Abraham and Barker [31] predicts the form of $g_{0}$,

$$
\begin{array}{r}
g_{0}(\zeta ; \nu)=\frac{1+2 \nu}{(1-\nu)^{2}} \\
-\frac{3}{5} \nu \frac{10-2 \nu+\nu^{2}}{1+2 \nu} \alpha_{1} \zeta+\frac{1}{5} \nu\left(3 \theta_{1}+2 \theta_{2}\right) \\
+12 \nu \int_{0}^{\zeta} \zeta^{\prime}\left[\alpha_{1}\left(\zeta^{\prime}-\zeta\right)+\theta_{1}+\theta_{2}\right] h_{11}\left(\zeta^{\prime}\right) d \zeta^{\prime} \\
+12 \nu \int_{\zeta}^{1+\zeta} \zeta^{\prime}\left(\zeta+1-\zeta^{\prime}\right)^{3}\left[\theta_{1}+\theta_{2}\left(\zeta+1-\zeta^{\prime}\right)\right] \\
h_{11}\left(\zeta^{\prime}\right) d \zeta^{\prime},
\end{array}
$$

where $h_{11}\left(\zeta^{\prime}\right) \equiv g_{11}\left(\zeta^{\prime}\right)-1, \alpha_{1} \equiv(1+2 \nu)^{2} /(1-$ $\nu)^{4}, \theta_{1} \equiv-2 \nu(1+\nu / 2)(1+2 \nu) /(1-\nu)^{4}$, and $\theta_{2} \equiv \nu \alpha_{1} / 2$. The function $g_{11}\left(\zeta^{\prime}\right)$ is the PY radial distribution function for a hard sphere, where 
$1 \leqslant \zeta^{\prime}<+\infty$ is the relative distance between sphere centers [30]. We calculate integrals in Eq. (27) numerically and, for convenience, prepare an interpolated look-up table for $g_{0}$ in terms of $\zeta$ and $\nu$.

We then calculate two quantities affecting heat transfer. The first is the fraction $\tilde{\nu}$ of the local volume that is occupied by solid material. To find it, we note that a sphere with center at $\zeta^{\prime}=y^{\prime} / d-1 / 2$ contributes a volume $\pi d^{3}\left[(1 / 4)-\left(\zeta-\zeta^{\prime}\right)^{2}\right] d \zeta$ to the parallelepiped or "strip" of thickness $d \times d \zeta$ and surface area $S$ parallel to the wall with $\left|\zeta-\zeta^{\prime}\right|<1 / 2$. Therefore, by summing this volume over the number of spheres $d N^{\prime}$ in Eq. (26) with $\zeta^{\prime} \in\left[\zeta^{\prime}, \zeta^{\prime}+d \zeta^{\prime}\right]$, and upon dividing by the strip volume $S d \zeta \times d$, we find the local oscillating "mass-averaged" solid volume fraction at a given distance $y=(\zeta+1 / 2) \times d$ from the wall

$$
\begin{array}{r}
\tilde{\nu}(\zeta)=\int_{\zeta^{\prime}=\zeta-1 / 2}^{\zeta+1 / 2} \frac{d N^{\prime} \pi d^{3}\left[(1 / 4)-\left(\zeta^{\prime}-\zeta\right)^{2}\right] d \zeta}{S d \zeta \times d} \\
\equiv \nu I_{\nu}(\zeta ; \nu),
\end{array}
$$

where the integral

$$
\begin{array}{r}
I_{\nu}\left(\frac{y}{d} ; \nu\right) \equiv 6 \int_{\zeta^{\prime}-\zeta=-1 / 2}^{+1 / 2}\left[\frac{1}{4}-\left(\zeta^{\prime}-\zeta\right)^{2}\right] \times \\
g_{0}\left(\zeta^{\prime} ; \nu\right) d\left(\zeta^{\prime}-\zeta\right)
\end{array}
$$

tends to unity as $y / d \rightarrow \infty$.

The second oscillating quantity is the available heat exchange surface area per unit volume. We note that a sphere with center at $\zeta^{\prime}=y^{\prime} / d-1 / 2$ contributes $\pi d / S$ to that quantity in the strip at $\zeta$. Then, the total exchange surface area per unit volume is

$$
\widetilde{n A}=\int_{\zeta^{\prime}=\zeta-1 / 2}^{\zeta+1 / 2}\left(\frac{\pi d}{S}\right) d N^{\prime} \equiv\left(\frac{6 \nu}{d}\right) I_{a}\left(\frac{y}{d} ; \nu\right),
$$

where the integral

$$
I_{a}\left(\frac{y}{d} ; \nu\right) \equiv \int_{\zeta^{\prime}-\zeta=-1 / 2}^{+1 / 2} g_{0}\left(\zeta^{\prime} ; \nu\right) d\left(\zeta^{\prime}-\zeta\right)
$$

also tends to unity as $y / d \rightarrow \infty$.

We evaluate the integrals $I_{\nu}$ and $I_{a}$ numerically with Mathematica for $y>0$ subject to $\zeta^{\prime}>$
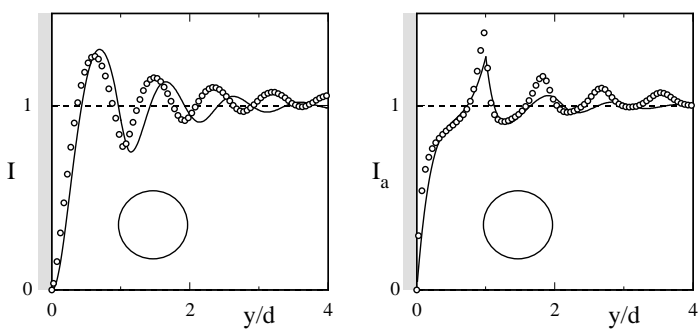

Figure 2. Dimensionless integrals $I_{\nu}$ (left) and $I_{a}$ versus $y / d$. The large circle represents the size of a sphere, the darkened left axis marks the position of the wall, and the dashed horizontal line is unity. Symbols are DEM simulation results collected in strips perpendicular to $y$. The lines are predictions of Eqs. (29) and (31). Conditions are $\nu=39 \%, L / d=8.7$ and $a_{0} / d=0.1$.

0 . Typically, direct evaluation of these integrals converges well up to $y / d \sim 4$. We substitute $\widetilde{n A}$ for $n A$ in Eq. (3) to capture the oscillating source term $\tilde{H}$ near the wall. For simplicity, we also assume that $\tilde{\nu}$ may be substituted for $\nu$ in Eq. (4) to predict the spatial variations $\tilde{K}_{g}$ of the local mixture thermal conductivity,

$$
\tilde{K}_{g}(y / d ; \nu)=K_{g}\left[\nu I_{\nu}(y / d ; \nu)\right] .
$$

Figure 2 compares the predictions of Eqs. (29) and (31) for $I_{\nu}$ and $I_{a}$ with Discrete-ElementModeling (DEM) numerical simulations of spheres agitated in a semi-infinite cubic domain with sinusoidal vibrations in the direction $x$ at the amplitude $a_{0}$ against two flat walls separated by a distance $L$, possessing another two parallel flat walls at different thermal temperatures located at $y= \pm L / 2$, and having periodic boundary conditions separated by $L$ in the third cartesian direction $z$. These is no gravity in this instance. The DEM hard-sphere algorithm is described by Hopkins and Louge [32].

As Fig. 2 shows, even at relatively large solid volume fractions, Eqs. (29) and (30) capture effects of local ordering within the first two to three sphere diameters from the wall with sufficient relative accuracy to predict $\widetilde{n A}, \tilde{K}_{g}$ and $\tilde{H}$ where 
oscillations in these quantities matter. However, the theory predicts oscillations in $I_{\nu}$ and $I_{a}$ that are increasingly out of phase with DEM simulations as $y / d$ grows.

\subsection{Source term}

A difficulty with grains agitated in a fluid with thermal temperature gradients is that they are subject to a complicated surface temperature spatial distribution and time-history, even in stationary systems. The grains can also exhibit internal temperature gradients, unless their Biot number vanishes. In this context, it is unclear a priori whether the source term is captured by Eq. (3) under any circumstances. To address this question, we model $H$ by summing the contribution of all grains in a unit volume to the heat exchanged between an individual sphere and the surrounding fluid. We calculate the exchange using the classical solution for unsteady conduction within a single sphere immersed in an infinite fluid. We then conduct simple thermal numerical simulations to evaluate the merits of this approach.

We model agitated grains immersed in a fluid as thermally independent spheres adopting an initial temperature $T_{s}$ upon an impact, and subsequently exchanging heat with a fluid of known bulk temperature $T_{g}$, at a constant Nusselt number, and on a time scale that is the inverse of the impact frequency

$$
f_{c}=\frac{24}{\sqrt{\pi}} \nu g_{12} \frac{\sqrt{\Theta}}{d} .
$$

In this view, we write the heat exchanged in unit volume and time as

$$
\tilde{H}=n I_{a}\left(\frac{y}{d} ; \nu\right) \frac{d Q}{d t},
$$

where $I_{a}(y / d ; \nu)$ is found in Eq. (31). $d Q / d t$ is calculated from the classical series solution for unsteady conduction in a sphere with cumulative heat $Q$ transferred to the fluid [33],

$$
\left(\frac{1}{Q_{0}}\right) \frac{d Q}{d \mathrm{Fo}}=6 \sum_{i=1}^{\infty} \frac{\exp \left(-\xi_{i}^{2} \mathrm{Fo}\right)}{\left[1-(1 / \mathrm{Bi})+\left(\xi_{i} / \mathrm{Bi}\right)^{2}\right]} .
$$

In this expression, the Fourier number based on sphere radius renders time dimensionless, Fo $\equiv$
$4 k_{s} t / \rho_{s} c_{s} d^{2}$, and the total heat eventually transferred is $Q_{0}=(\pi / 6) d^{3} \rho_{s} c_{s}\left(T_{s}-T_{g}\right)$. The eigenvalues $\xi_{i}$ are solutions of the equation $\xi / \tan \xi=$ $1-\mathrm{Bi}$. To evaluate $\tilde{H}$, we adopt a Fourier number

$$
\mathrm{Fo}_{c}=\frac{4 k_{s}}{f_{c} \rho_{s} c_{s} d^{2}}
$$

based on the grain mean free time $1 / f_{c}$. Combining Eqs. (34) and (35) with definitions of $\mathrm{Nu}$ and $\mathrm{Bi}$, the source term becomes

$$
\begin{array}{r}
\tilde{H}=12 \nu \frac{k_{g}}{d^{2}} \mathrm{Nu}\left(T_{s}-T_{g}\right) I_{a}\left(\frac{y}{d} ; \nu\right) \times \\
\sum_{i=1}^{\infty} \frac{2 \exp \left(-\xi_{i}^{2} \mathrm{Fo}_{c}\right)}{\left[\mathrm{Bi}-1+\xi_{i}^{2} / \mathrm{Bi}\right]} .
\end{array}
$$

Unless the Fourier number is very large, it is sufficient to retain only the first term in the series. For $\mathrm{Bi}<6$, the first eigenvalue is approximated to a relative error $<1 \%$ by $\xi_{1}=$ $\sqrt{3 \mathrm{Bi}}\left[1-\mathrm{Bi} / 10+\mathrm{Bi}^{2} / 156+o\left(\mathrm{Bi}^{3}\right)\right]$, where the term $\propto \mathrm{Bi}^{5 / 2}$ is inexact. Then, the source term is approximated to better than $1 \%$ for $\mathrm{Bi}<1.3$ with

$$
\begin{array}{r}
\tilde{H} \approx 12 \nu \frac{k_{g}}{d^{2}} \mathrm{Nu}\left(T_{s}-T_{g}\right) I_{a}\left(\frac{y}{d} ; \nu\right) \exp \left(-\xi_{1}^{2} \mathrm{Fo}_{c}\right) \times \\
{\left[1-\frac{\mathrm{Bi}}{5}+\frac{3}{520} \mathrm{Bi}^{2}+\frac{99}{13000} \mathrm{Bi}^{3}+o\left(\mathrm{Bi}^{4}\right)\right],}
\end{array}
$$

where terms of order higher than $\mathrm{Bi}$ are inexact. In the limit of vanishing $\mathrm{Bi}$, and far enough away from the wall, Eq. (38) reduces to Eq. (3).

Because $\tilde{K}_{g}$ and $\tilde{H}$ oscillate with distance from the thermal walls, it is no longer possible to find an analytical solution of Eqs. (1) and (2) subject to boundary conditions (11) and (12). Instead, we employ MATLAB's two-point boundary value solver bvp4c, in which we calculate $I_{a}(y / d ; \nu)$ and $I_{\nu}(y / d ; \nu)$ by interpolating look-up tables for $0 \leqslant y / d \leqslant 4$ and $0.001 \leqslant \nu \leqslant 0.5$ and, for simplicity, by truncating oscillations at large $y / d$ setting $I_{a}=I_{\nu}=1$ for $y / d>4, \forall \nu$.

\subsection{Thermal simulations}

In this section, we incorporate simple heat balances in the DEM simulations to test the form of the source term $\tilde{H}$ in Eq. (37) and the role of $\tilde{K}_{g}$. Figure 3 illustrates the partitioning of space used for thermal balances in these simulations. 


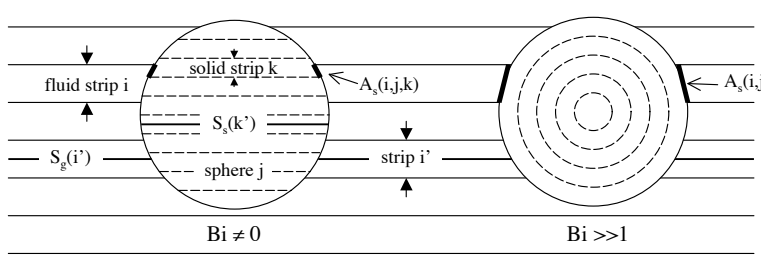

Figure 3. Partitioning of fluid and spheres in thermal simulations. The space around the spheres is subdivided in identical "fluid strips" of uniform $T_{g}$ parallel to the two flat thermal walls at temperatures $T^{+}$and $T^{-}$. The fluid strip $i^{\prime}$ has a cross-section $S_{g}\left(i^{\prime}\right)$ that excludes solid material. Spheres of finite Biot numbers (left) are subdivided in equal "solid strips" of uniform $T_{s}$ perpendicular to the imposed mean temperature gradient and typically thinner than fluid strips. The cross-section of solid strip $k^{\prime}$ is $S_{s}\left(k^{\prime}\right)$. The axisymmetric exchange surface area between fluid strip $i$ and solid strip $k$ of sphere $j$ is $A_{s}(i, j, k)$. Because spheres move, this area is updated at each DEM time step. Spheres with $\mathrm{Bi}=0$ have uniform temperature $T_{s}$ and thus possess a single solid "strip," $k=1$. Spheres of high Bi (right) are modeled as concentric shells of identical thickness and uniform temperature; the exchange surface area between the outer shell of sphere $j$ and fluid strip $i$ is $A_{s}(i, j)$. It is also updated at every DEM time step.
The gas temperature profile is obtained by solving the one-dimensional transient heat conduction equation on fluid strips at times imposed by the DEM simulation; in continuous form it is

$$
\rho_{g} c_{g} \frac{\partial T_{g}}{\partial t}=-\frac{1}{S_{g}} \frac{\partial}{\partial y}\left(-k_{g} S_{g} \frac{\partial T_{g}}{\partial y}\right)+H,
$$

where $\rho_{g}$ and $c_{g}$ are, respectively, the density and specific heat per mass of the fluid; $S_{g}(y, t)$ is the cross-section area of planes at constant $y$ that is occupied by the fluid at time $t$ (Fig. 3). Its complement is the area intersected by spheres. In the discrete form of Eq. (39), the volumetric rate of heat added to fluid strip $i$ is

$$
H(i)=\dot{q}_{s}(j, k) / V_{g}(i),
$$

where

$\dot{q}_{s}(j, k)=\sum_{j \in i} \sum_{k \in i} h A_{s}(i, j, k)\left[T_{s}(j, k)-T_{g}(i)\right]$

is the rate of heat given by solid strip $k$ of sphere $j$ to fluid strip $i$. In Eqs. (40) and (41), $j \in i$ indicates all spheres intersecting fluid strip $i, k \in i$ refers to all solid strips of sphere $j$ intersecting fluid strip $i, V_{g}(i)$ is the fluid volume within strip $i, h=k_{g} \mathrm{Nu} /(d / 2)$ is the heat transfer coefficient, $T_{s}(j, k)$ is the uniform temperature of solid strip $k$ within sphere $j, T_{g}(i)$ is the uniform fluid temperature of strip $i$, and $A_{s}(i, j, k)$ is the part of the external surface area of particle $j$ that resides in strip $i$ and intersects solid strip $k$, see Fig. 3 (left). We employ the Tri-Diagonal Matrix Algorithm (TDMA) [34] to solve Eq. (39) subject to the prescribed gas temperatures $T^{+}$and $T^{-}$at opposite thermal walls.

Knowing $T_{g}(i)$, we use the TDMA again to solve at each DEM time step the energy balance within each sphere $j$ of moderate Biot number subject to $\left.\left(\partial T_{s} / \partial y\right)\right|_{y=y_{c} \pm d / 2}=0$ at both poles of $j$ centered at $y_{c}(j)$. In continuous form, the sphere energy balance is

$$
\begin{array}{r}
\rho_{s} c_{s} \frac{\partial T_{s}(j)}{\partial t}=-\frac{1}{S_{s}(j)} \frac{\partial}{\partial y}\left(-k_{s} S_{s}(j) \frac{\partial T_{s}(j)}{\partial y}\right) \\
-\dot{q}_{s}(j),
\end{array}
$$

where $\dot{q}_{s}(j)$ is given by Eq. (41) in discrete form and $S_{s}(j ; y, t)$ is the cross-section area of sphere $j$ 
cut by the plane of constant $y$ at time $t$ (Fig. 3). In Eq. $(42), T_{s}(j)$ and $\dot{q}_{s}(j)$ both vary with $y$ within sphere $j$ unless its Biot number vanishes. If it does, then sphere $j$ with $\mathrm{Bi}=0$ is no longer subdivided in solid strips, but instead has a uniform temperature $T_{s}(j)$.

For spheres of large Biot number, interior conduction is mostly radial. Accordingly, we model it by dividing the sphere in concentric shells of equal thickness and uniform temperature (Fig. 3, right). We use again the TDMA to solve the heat conduction equation, which is, in continuous form

$$
\rho_{s} c_{s} \frac{\partial T_{s}(j)}{\partial t}=-\frac{1}{r^{2}} \frac{\partial}{\partial r}\left(-k_{s} r^{2} \frac{\partial T_{s}(j)}{\partial r}\right),
$$

subject to symmetry at the sphere's center

$$
\left.\frac{\partial T_{s}(j)}{\partial r}\right|_{r=0}=0,
$$

and to the surface boundary condition

$$
\begin{array}{r}
-\left.\left(\pi d^{2}\right) k_{s} \frac{\partial T_{s}(j)}{\partial r}\right|_{r=d / 2}= \\
\sum_{i \in j} h A_{s}(i, j)\left[T_{s}(j ; r=d / 2, t)-T_{g}(i)\right],
\end{array}
$$

where $i \in j$ denotes all fluid strips wetting sphere $j$, and $A_{s}(i, j)$ is the axisymmetric part of the external surface area of particle $j$ that resides in strip $i$, see Fig. 3 (right).

To reduce the computation time needed to reach steady state thermal profiles, we start all simulations with an initial $T_{g}$ varying linearly between the two thermal walls at $T^{+}$and $T^{-}$, and with a uniform $T_{s}=\left(T^{+}+T^{-}\right) / 2$. Once steadystate has been reached, because $T_{g}(i)$ is effectively an average over the entire fluid strip $i$, it fluctuates little with time despite particle agitation. We map its counterpart for solids $\bar{T}_{s}$ on the same fluid strip $i$ by summing the sensible energy that each sphere contributes to the strip,

$$
\begin{gathered}
\bar{T}_{s} \sum_{j \in i} \sum_{k \in i} \rho_{s} c_{s} V_{s}(i, j, k)= \\
\sum_{j \in i} \sum_{k \in i} \rho_{s} c_{s} V_{s}(i, j, k) T_{s}(j, k),
\end{gathered}
$$

where $V_{s}(i, j, k)$ is the sphere volume between the two latitudes delimited by $A_{s}(i, j, k)$ for $\mathrm{Bi} \neq 0$ or $A_{s}(i, j)$ for $\mathrm{Bi} \gg 1$.
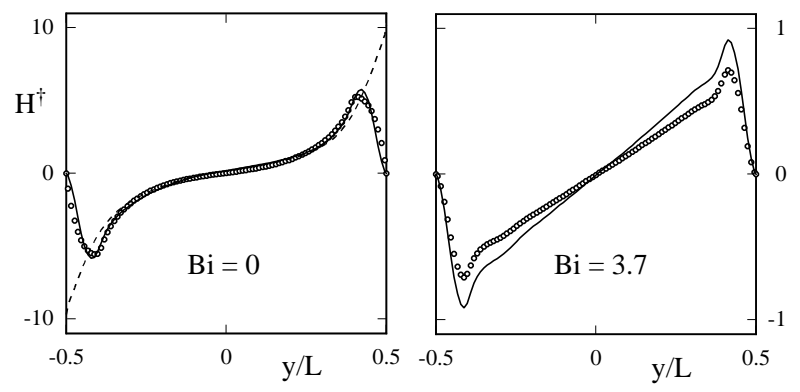

Figure 4. Dimensionless source term $H^{\dagger}$ vs. $y / L$. Circles are computed from thermal simulations using Eq. (40) and 99 fluid strips; the dashed line on the left is the prediction of Eq. (3) without spatial variations of $\nu$ (it clearly fails near the walls); the solid line is the prediction of Eq. (37) with actual oscillations $\tilde{\nu}$ in $\tilde{K}_{g}$ and $\widetilde{n A}$ in $\tilde{H}$. In Eq. (37), it is sufficiently accurate to retain only the first term in the series. Conditions are $\nu=10 \%, L / d=9.7, a_{0} / d=0.1$, and $\mathrm{Nu}=1$. Left graph: $\mathrm{Bi}=0$ (uniform temperature imposed within each sphere consistent with the high solid conductivity $\left.k_{s} / k_{g}=5900\right)$ and $\overline{\text { Fo }}=0.95$; in the bulk, this leads to a relatively low local Fourier number Fo $\approx 0.1, K_{s} / K_{g} \approx 57$, and $K_{g} / k_{g} \approx 1.3$. Right: simulations using the method in Fig. 3 (right) with 10 shells; conditions are $\mathrm{Bi}=3.7$ and $\overline{\mathrm{Fo}}=0.57$, with Fo $\approx 0.1, K_{s} / K_{g} \approx 94$, and $K_{g} / k_{g} \approx 0.91$.

In addition to the relative vibration amplitude $a_{0} / d$, the relative box size $L / d$, its geometrical aspect ratios, $\nu$, and impact parameters, thermal simulations are characterized by three dimensionless numbers, namely the particle Nusselt and Biot numbers, and a global Fourier number based on vibration frequency $f, \overline{\text { Fo }} \equiv 4 k_{s} / f \rho_{s} c_{s} d^{2}$.

Figure 4 shows the resulting source term, made dimensionless using $H^{\dagger} \equiv H d^{2} /\left[k_{g}\left(T^{+}-T^{-}\right) \nu\right]$. At high Biot number (Fig. 4, right), it is important to include the series correction term in Eq. (37), generally truncated to first order, to predict $H^{\dagger}$ accurately. For this correction, the appropriate Fourier number is based on local mean 
free time, see Eq. (36). However, $H^{\dagger}$ hardly matters to heat transfer at the wall when $\mathrm{Bi} \gg 1$ : because $H^{\dagger} \propto 1 / \mathrm{Bi}$ is low, the thermal temperatures of fluid and solids are nearly decoupled. Thus, the fluid temperature has a nearly straight profile between opposite walls, irrespective of particle agitation or Damköhler number. Similarly, if the spheres have the slightest agitation, their selfdiffusion ensures that $T_{s}$ is independent of $y / L$. Effectively, $L^{\dagger} \rightarrow 0$ as $\mathrm{Bi} \rightarrow \infty$. Thus, consistent with Eq. (19), $q / q_{0} \approx K_{g} / k_{g}$. Therefore, solids with $\mathrm{Bi} \gg 1$ do not enhance the thermal flux at the wall. Instead, because $k_{s} / k_{g}=\mathrm{Nu} / \mathrm{Bi}<1$, they typically reduce the mixture thermal conductivity, $K_{g}<k_{g}$.

At low Biot numbers, the series term in Eq. (37) approaches unity. This equation predicts $\tilde{H}$ accurately again, as long as spatial oscillations are considered with $I_{a}(y / d ; \nu)$, see Fig. 4 (left). As the dashed line in Fig. 5 shows, failing to capture $I_{a}$ properly, for example by adopting Eq. (3), over-estimates $\tilde{H}$ at the wall, where the thermal exchange between fluid and solids matters most. In the exchange limit, Eq. (22) indicates that the flux ratio scales as $q / q_{0} \propto H^{1 / 2} \propto \mathrm{Nu}^{1 / 2}$. Therefore, in this limit, the role of spatial oscillations in reducing $q / q_{0}$ is conveniently captured by an effective Nusselt number

$$
\begin{aligned}
\mathrm{Nu}_{\mathrm{eff}} \equiv \mathrm{Nu} \times & {\left[\lim _{K_{s} / K_{g} \rightarrow \infty}\left(\frac{\tilde{q}}{q_{0}}\right)\right]^{2} / } \\
& {\left[\lim _{K_{s} / K_{g} \rightarrow \infty}\left(\frac{q}{q_{0}}\right)\right]^{2}, }
\end{aligned}
$$

which may be substituted for $\mathrm{Nu}$ in Eq. (16) to predict $\tilde{q} / q_{0}$ analytically from Eq. (19) without computing $I_{\nu}$ or $I_{a}$. In Eq. (47), which defines $\mathrm{Nu}_{\text {eff }}, \tilde{q}$ denotes the flux calculated with spatial oscillations in $I_{\nu}$ and $I_{a}$, and $q$ without. To evaluate $\mathrm{Nu}_{\text {eff }}$, we integrate Eqs. (1) and (2) numerically as mentioned in the last paragraph of section 3.2 .

Figure 6 shows how $\mathrm{Nu}_{\text {eff }} / \mathrm{Nu}$ varies with $\nu$. In the range $0 \leqslant \nu \leqslant 0.5$ and $L / d \geqslant 5$, the following expression conveniently captures this prediction to a relative error $<2 \%$ :

$$
\frac{\mathrm{Nu}_{\mathrm{eff}}}{\mathrm{Nu}} \approx \frac{1+\nu\left(1-e^{-L / L_{0}}\right)\left(b_{\nu}+a_{\nu} \nu\right)}{1+\nu\left(1-e^{-L / L_{0}}\right)\left(d_{\nu}+c_{\nu} \nu\right)},
$$

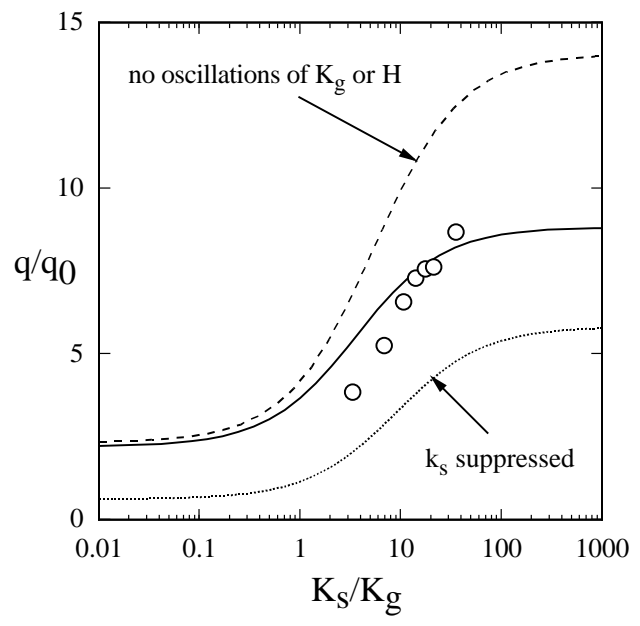

Figure 5. Flux ratio $q / q_{0}$ versus $K_{s} / K_{g}$ for $\mathrm{Nu}$ $=1, k_{s} / k_{g}=5900, L / d=9.7$, and $\nu=30 \%$. Circles are thermal simulations; for these data, $K_{s}$ and $K_{g}$ are evaluated with the average granular temperature $\Theta$ and volume fraction $\nu$ in the cell, using Eqs. (7) and (4), respectively. The top dashed line is the prediction of Eq. (19), in which wall-induced spatial oscillations are ignored. This clearly fails. The bottom dotted line is a model in which spatial oscillations derive from the HAB theory of Eqs. (27) to (31), but where heat is artificially barred from conducting through the spheres, $k_{s}=k_{g} \xi_{s}=0$ in Eq. (4), despite Bi $\ll 1$. This fails as well. The solid line is our recommended model, which incorporates spatial oscillations of $I_{a}$ in $\tilde{H}$ and the mixture conductivity from Eqs. (4) and (32). Such oscillations make it necessary to solve Eqs. (1) and (2) numerically. The upper bound of simulated $K_{s} / K_{g}$ is limited by computation time, which grows with $f, f_{c}, \overline{\mathrm{Fo}}^{-1}$, or $K_{s}$. 


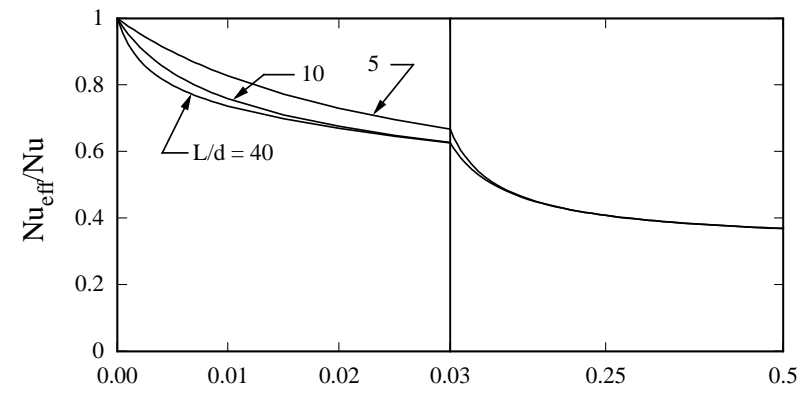

Figure 6. Effective Nusselt number capturing the effects of spatial oscillations versus bulk solid volume fraction. The abrupt change of abscissa at $\nu=0.03$ is meant to highlight the dependence of $\mathrm{Nu}_{\text {eff }}$ on $L / d$ at low $\nu$; such dependence is continuous.

where $L_{0} / d \approx 12.8, a_{\nu} \approx 1385, b_{\nu} \approx 230, c_{\nu} \approx$ 4370, and $d_{\nu} \approx 327$. Spatial oscillations reduce $\mathrm{Nu}_{\text {eff }}$ at remarkably low $\nu$. Nonetheless, with a mixture conductivity given by Eq. (4), the wall heat transfer is always enhanced at vanishing Biot number, $\forall \nu$. This is because, to lowest order, the flux ratio rises with $\nu$ and $L / d>5$ as $\tilde{q} / q_{0}=$ $1+\left[3+\left(b_{\nu}-d_{\nu}\right)\left(1-e^{-L / L_{0}}\right) / 2+\mathrm{Nu}(L / d)^{2}\right] \nu+$ $o\left(\nu^{2}\right)>1$.

Although $\mathrm{Nu}_{\text {eff }}$ was meant to capture $\tilde{q} / q_{0}$ analytically for $\mathrm{Bi} \ll 1$ in the exchange limit $\left(K_{s} / K_{g} \rightarrow \infty\right.$, or $\left.\mathrm{Da} \rightarrow 0\right)$, it is also a good approximation for any Damköhler ratio. For example, substituting $\mathrm{Nu}_{\text {eff }}$ from Eq. (48) for $\mathrm{Nu}$ in Eq. (16) yields a flux ratio $\tilde{q} / q_{0}$ calculated from Eq. (19) with a relative error $<4 \%$ at $\nu=0.01$ and $<9 \%$ at $\nu=0.5$.

As Fig. 5 illustrates, the wall heat flux is affected by gradient-driven conduction through solid spheres. If such conduction was suppressed by making $\xi_{s}=0$ in Eq. (4), while artificially keeping $\mathrm{Bi} \ll 1$, then $\tilde{q} / q_{0}$ would be underpredicted, as the dashed line clearly shows. To capture this conduction within the spheres as simply as possible, we assume that it is driven by $\nabla T_{g}$ through the mixture conductivity (4) in the energy equation for the fluid. When spatial vari- ations of the mixture conductivity are taken into account by adopting Eq. (32), and when $\tilde{H}$ is allowed to oscillate near the wall through $I_{a}$ in Eq. (37), our relatively simple model agrees well with simulations (Fig. 5).

Nonetheless, the thermal simulations are crude for several reasons. First, they ignore any coupling between particle and gas velocities, and possible effects of the latter on convective heat transfer between fluid and solids. Instead, they adopt a constant heat transfer coefficient independent of local solid volume fraction and particle-induced gas velocity fluctuations. Then, they simplify the three-dimensional conjugate fluid-solid heat transfer problem by privileging a gradient direction in each sphere, and by ignoring fluid temperature gradients perpendicular to the $y$-axis.

Despite these simplications, they confirm that the local gradient $\nabla T_{g}$ and the local temperature difference $\left(T_{s}-T_{g}\right)$ simultaneously play a role in setting the overall heat transfer flux. In particular, they show that, in addition to the exchange carried by $\left(T_{s}-T_{g}\right)$ through the locally available exchange surface area, the gradient also drives a heat flux through the spheres that is well captured by homogenization models, as long as the solid volume fraction is allowed to oscillate in response to spatial ordering from the flat thermal walls. At large Biot, they also provide a first taste of the role of thermal history by revealing that the appropriate Fourier number is based on the mean time of flight of the colliding spheres.

In the next sections, we attempt to refine our description of the heat transfer between fluid and solid by adopting simple corrections to the heat transfer coefficient $h$, and by correcting the homogenized mixture conductivity with turbulent enhancements driven by particle agitation. We will describe the experiments that elicited these refinements in Part II.

\subsection{Enhancements at high agitation}

We apply the present analysis to situations in which particle agitation is not derived from fluid velocity fluctuations. This is the case, for example, when relatively dense suspensions of massive particles owe their fluctuation velocity to repeated collisions. Such particles have a Stokes 
number St $\equiv \rho_{s} d^{2} / 18 \mu \tau \ggg 1$, where $\mu$ is the fluid's dynamic viscosity and $\tau$ is a characteristic time of the flow. We also restrict attention to situations without a mean relative velocity between fluid and solids.

For massive particles in a gas at relatively large solid volume fractions $\left(\rho_{s} \gg \rho_{g}, \nu>0.05\right)$, it is challenging to test our model with experiments in the presence of gravitational accelerations. To defeat gravity without imposing a relative velocity between gas and solids, considerable agitation must be given to the particles. Although massive particles with St $\gg 1$ are not affected by gas velocity fluctuations, their agitation can induce such fluctuations in their wakes, thus augmenting the mixture conductivity $K_{g}$ in Eqs. (4) and (32), and raising the conductivity on which the Nusselt number in Eq. (3) is based. An understanding of these effects requires a detailed model of gas-solid interactions. In dilute turbulent suspensions, much progress has been made on twoway coupling between gas and solids, which can either enhance or reduce turbulent gas velocity fluctuations, and ultimately augment $k_{g}$ and $K_{g}$ [35-41]. In the experiments described in Part II, the suspension is too dense $(\nu>0.06)$ and the Stokes number too large $(430<\mathrm{St}<16,000)$ for us to adopt this existing turbulent framework.

A suitable model for the thermal exchange between grain and gas must simultaneously account for the effects of high solid volume fraction and for a surrounding turbulence that is not generated by a mean relative velocity between solids and gas. Thus, in contrast to the dilute "riser" flows considered by Louge, et al [18], we do not expect correlations like Whitaker's [42], which correct the infinite-fluid, pure-conduction result $\mathrm{Nu}=1$ for small relative velocity between an isolated particle at rest and a steady gas flow, to capture the role of high agitation on the source term by increasing $\mathrm{Nu}$ with a particle Reynolds number based on $\sqrt{\Theta}$. Instead, as outlined next, we adopt $\mathrm{Nu}=1$, account for gas velocity fluctuations by augmenting the conductivity on which $\mathrm{Nu}$ is based, and capture the role of high solid volume fraction through the mixture conductivity. The experiments in Part II will test the merit of this approach.

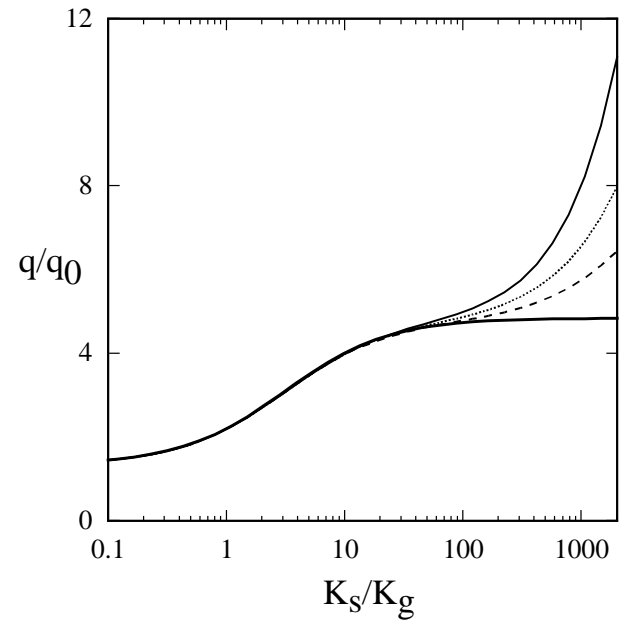

Figure 7. Relative importance of augmentation mechanisms of $q / q_{0}$ with high agitation. Here we assume uniform $\Theta$ and $\nu$, but integrate Eqs. (1) and (2) numerically using oscillating $I_{\nu}$ and $I_{a}$ from Eqs. (29) and (31) near the walls. The abscissa is $K_{s} / K_{g}$, where $K_{g}$ is the static mixture conductivity given by Eq. (4) at the bulk solid volume fraction $\nu=0.195$. Conditions are $\operatorname{Pr}=0.7$, $\rho_{g} c_{g} / \rho_{s} c_{s}=7 \times 10^{-4}, k_{s} / k_{g}=5$, and $L / d=7.9$, corresponding to $3.2 \mathrm{~mm}$ acrylic spheres in a box of $L=25.4 \mathrm{~mm}$ filled with air. From top to bottom: the thin solid line marks the recommended model, which substitutes $K_{g t}$ for $K_{g}$, bases the Nusselt number on $k_{g t}$, and implements the BiotFourier correction of Eq. (38); the dotted line bases $\mathrm{Nu}$ on $k_{g}$ instead, but still substitutes $K_{g t}$ for $K_{g}$; the dashed line uses $K_{g}$, but bases $\mathrm{Nu}$ on $k_{g t}$; the thick horizontal asymptote ignores all augmentation mechanisms $\left(k_{t}=0\right)$ and takes $\mathrm{Bi}=0$. Under these conditions, high-agitation augmentation mechanisms become significant at $K_{s} / K_{g}>65$, where $\operatorname{Re}>4.5$ and $\mathrm{St}>1900$. 
Verberg and Koch [43] conducted LatticeBoltzmann numerical simulations of spheres sheared a semi-infinite domain between two bumpy boundaries to refine the expressions of Sangani, et al $[7,8]$ for the volumetric rate of dissipation of granular fluctuation energy

$$
\gamma_{\text {vis }}=\frac{54 \nu \mu \Theta}{d^{2}} R_{\text {diss }}\left(\nu, \epsilon_{m}, \operatorname{Re} / \sqrt{3}\right)
$$

by providing the coefficient $R_{\text {diss }}$ in terms of $\nu$, a parameter $\epsilon_{m}=9.76 \lambda_{g} / d$ accounting for noncontinuum lubrication between colliding spheres in a gas of molecular mean free path $\lambda_{g}$, and a Reynolds number based on $\sqrt{\Theta}$ and $d$. Combining Eqs. (5) and (7), this particle Reynolds number is

$$
\begin{array}{r}
\frac{\operatorname{Re}}{\sqrt{3}}=\frac{9 \sqrt{\pi} g_{12}}{\operatorname{Pr}}\left(\frac{\rho_{g} c_{g}}{\rho_{s} c_{s}}\right) f_{M}\left(\nu ; \xi_{s}\right) \times \\
\left(\frac{K_{s}}{K_{g}}\right)(1+2 \mathrm{Kn}) \mathrm{Le}_{s},
\end{array}
$$

where $f_{M}$ is defined in Eq. (4) and is evaluated at the bulk $\nu$.

Verberg and Koch [43] also measured the dimensionless Reynolds stress $-\overline{u_{i}^{\prime} u_{j}^{\prime}} /\left[d \Gamma_{u} / 2\right]^{2}$ and granular temperature $\sqrt{\Theta} /\left[d \Gamma_{u} / 2\right]$, where $u_{i}^{\prime}$ is the gas fluctuation velocity in the cartesian direction $i$ and $\Gamma_{u}$ is the applied strain rate. They found that both quantities are proportional to the ratio $\mathrm{St}_{\gamma} / R_{\text {diss }}$, where $\mathrm{St}_{\gamma} \equiv \rho_{s} d^{2} \Gamma_{u} / 18 \mu$, so the Reynolds stress scales as

$$
\rho_{g} \overline{u_{i}^{\prime} u_{j}^{\prime}}=-\omega \rho_{g} \Theta^{1 / 2}\left(\frac{\partial u_{i}}{\partial x_{j}}+\frac{\partial u_{j}}{\partial x_{i}}\right) d,
$$

where $\omega$ is a constant expected to depend on $\nu$; they reported $\omega=0.037(+0.014,-0.010)$ at $\nu=0.3$ with $i$ and $j$ in the flow and gradient directions, respectively.

To model the augmentation of $k_{g}$ by gas velocity fluctuations, we invoke "Reynolds' analogy" and write

$$
k_{g t}=k_{g}+k_{t},
$$

where

$$
\left(\frac{k_{t}}{\rho_{g} c_{g}}\right)=\frac{\left(\mu_{t} / \rho_{g}\right)}{\operatorname{Pr}_{t}}
$$

is the additional heat diffusivity induced by gas velocity fluctuation, $\mathrm{Pr}_{t} \simeq 0.9$ is a turbulent Prandtl number [18], and $\mu_{t}$ is an eddy viscosity defined as

$$
\rho_{g} \overline{u_{i}^{\prime} u_{j}^{\prime}}=-\mu_{t}\left(\frac{\partial u_{i}}{\partial x_{j}}+\frac{\partial u_{j}}{\partial x_{i}}\right) .
$$

Combining Eqs. (51), (53) and (54), and eliminating $\Theta$ in terms of $K_{s}$ using Eqs. (5) and (7), we find

$$
\begin{array}{r}
\frac{k_{t}}{k_{g}}=\omega \frac{9 \sqrt{\pi} \mathrm{Le}_{s}}{\operatorname{Pr}_{t}}\left(\frac{\rho_{g} c_{g}}{\rho_{s} c_{s}}\right) f_{M}\left(\nu ; \xi_{s}\right) g_{12} \times \\
(1+2 \mathrm{Kn})\left(\frac{K_{s}}{K_{g}}\right) .
\end{array}
$$

This equation reveals that, unless $\rho_{g} c_{g} / \rho_{s} c_{s}$ nearly vanishes (as it would, for example, under the low atmospheric pressure of Mars), solid velocity fluctuations affect heat transfer in the exchange limit by raising the conductivity of the gas to $k_{g t}$. In the limit of small solid volume fractions, Eq. (55) reduces to

$$
\lim _{\nu \rightarrow 0} \frac{k_{t}}{k_{g}}=\omega \frac{\sqrt{\Theta} d}{\operatorname{Pr}_{t}\left(k_{g} / \rho_{g} c_{g}\right)} .
$$

Because particle-induced gas transport coefficients should vanish as solids disappear, we expect that $k_{t} \rightarrow 0$ as $\nu \rightarrow 0$. Because $\Theta$ does not tend to zero in that limit, $\omega$ should vary with $\nu$, and vanish with it. In the absence of published data for $\omega(\nu)$, we assume $\omega \propto \nu$ and adopt the value that Verberg and Koch [43] measured at $\nu=0.3$ i.e., $\omega=\nu \times 0.037 / 0.3$. Our thermal measurements in Part II will test the merit of this simple expression for $\omega$.

Therefore, in our view, high particle agitation has three principal effects on the exchange limit. First, it increases the source term, so that $k_{g t}$ must be substituted for $k_{g}$ in Eq. (16) for $L^{\dagger}$ (but not in Eq. (19), where $k_{g}$ represents the molecular conductivity of pure gas). Second, the Biot number increases to $\left(k_{g t} / k_{s}\right) \mathrm{Nu}$, possibly leading to significant corrections of $\tilde{H}$ calculated with Eq. (38). Third, the mixture conductivity $K_{g}$ rises to a new $K_{g t}$, which we model as in Eq. (4),

$$
K_{g t}=k_{g t} f_{M}\left(\nu ; \xi_{s t}\right),
$$


where $\xi_{s t} \equiv k_{s} / k_{g t}$, and which we substitute wherever $K_{g}$ appears in Eqs. (1), (16), (18) and (19).

Near the wall, where $\nu$ oscillates, we replace $\tilde{K}_{g}$ by $\tilde{K}_{g t}$ as shown in Eq. (32). To account for these oscillations, we integrate once again Eqs. (1) and (2) numerically along $y$ with MATLAB's bvp4c using $k_{g t}$ and $K_{g t}$ instead of $k_{g}$ and $K_{g}$, respectively. Because gas velocity fluctuations diffuse on a length scale on the order of $d$, we ignore fluctuations of $\tilde{\nu}$ to evaluate $k_{t}$, but instead use the bulk solid volume fraction in Eq. (55). Figure 7 illustrates the results and shows the relative importance of raising $k_{g}$ to $k_{g t}$ and $K_{g}$ to $K_{g t}$. Notably, we find that numerical integration can be avoided by capturing spatial oscillations using the effective $\mathrm{Nu}_{\text {eff }}$ in Eq. (48), while substituting $k_{g t}$ for $k_{g}$ in Eq. (16) and $K_{g t}$ for $K_{g}$ in Eqs. (16) and (19). For conditions of Fig. 7, this approximation for $q / q_{0}$ has relative error $<8 \%$.

\subsection{Enhancement limitations}

In principle, enhancements of $q / q_{0}$ induced by high solids agitation rise ad infinitum with $K_{s} / K_{g}$. However, as the following calculation shows, collisional dissipation limits practical values of granular temperature, and thus $K_{s}$, which may be achieved away from boundaries that impart agitation on the grains. In Part II, we will describe a model for solids agitation in the vibrated box. We adopt a simpler approach here. In the absence of gravity, stress work or convection, the balance of fluctuation energy for nearly elastic, frictionless spheres experiencing instantaneous, binary collisions in a semi-infinite domain bounded by a wall of normal $z$ is

$$
0=-\frac{d}{d z}\left(-\kappa \frac{d \Theta}{d z}\right)-\gamma
$$

where $\kappa=(4 / \sqrt{\pi}) M \rho_{s} \nu^{2} g_{12} d \sqrt{\Theta}$ is the conductivity of granular fluctuation energy, $M=$ $1+(9 \pi / 32)\left[1+5 /\left(12 \nu g_{12}\right)\right]^{2}, \gamma=(12 / \sqrt{\pi})(1-$ $\left.e^{2}\right) \rho_{s} \nu^{2} g_{12} \Theta^{3 / 2} / d$ is the volumetric rate of collisional dissipation, and $0 \leqslant e<1$ is a dimensionless kinematic coefficient characterizing the incomplete post-impact restitution of the normal component of the relative velocity of two colliding grains [44]. (In our experiments of Part
II, the dissipation of granular fluctuation energy by the gas can be neglected in Eq. (58) i.e., $\left.\gamma_{\text {vis }} \ll \gamma\right)$. Defining the dimensionless distance $z^{*} \equiv z / d$ from an energetic wall at granular temperature $\Theta_{0}$ and the dimensionless fluctuation velocity $w^{*} \equiv \sqrt{\Theta / \Theta_{0}}$, and assuming an invariant $\nu$, Eq. (58) can be written $d^{2} w^{* 3} / d z^{* 2}=$ $9\left(1-e^{2}\right) w^{* 3} / 2 M$. Its solution $w^{*}=\exp \left[-z^{*} / \ell^{*}\right]$ decays from the wall on a dimensionless length scale $\ell^{*}=\sqrt{2 M /\left(1-e^{2}\right)}$, which decreases with denser $\nu$ and lower $e$. $\ell^{*}$ is relatively small for values of $e$ typical of real sphere materials. For example, at $\nu=5 \%$ and $e=0.9, \ell^{*} \approx 26$; at $\nu=30 \%$, it is down to $\ell^{*} \approx 6$.

Beyond a distance from the wall $z>d \times \ell^{*}$, the granular medium is unlikely to have sufficient agitation to augment $\tilde{K}_{g}$ and $k_{g}$, or perhaps to remain in the exchange limit. In practical situations, our thermal analysis must be coupled to granular mechanics equations describing the profiles of $\Theta$ and $\nu$ [9], which, for simplicity, we have taken to be constant in Part I, but will revisit in greater detail in Part II.

\section{Conclusions}

In Part I, we outlined a theory for the enhancement of heat transfer through a flat thermal wall bounding a homogeneous suspension of agitated spherical grains in a fluid. The theory is based on explicit hypotheses and independently measurable material parameters. Its crucial assumption is that grains do not exchange any thermal energy during their ephemeral contacts with each other or with the wall. However, their presence enhances heat transfer by modifying the mixture conductivity and by steepening the fluid temperature gradient at the wall. The steepening is the result of a competition between thermal selfdiffusion of the grains and their exchange of heat with the surrounding fluid, which is arbitrated by a Damköhler second ratio. Self-diffusion is a process that is not driven by a gradient of particle concentration, but rather by grain agitation. It gives rise to conduction through the solid phase if the latter possesses a thermal temperature gradient.

At high values of Da, which we call the "diffu- 
sion limit," the enhancement is governed by granular self-diffusion. The diffusion limit is relevant to small particles or low agitation. At low values of Da, which we call the "exchange limit," the volumetric rate of heat exchange dominates. We derived expressions for this exchange up to Biot numbers $\sim o(10)$, and tested these in crude, but instructive numerical simulations.

We showed that the ordering imposed by the flat thermal wall affects heat transfer by changing the local mixture conductivity and volume exchange rate between fluid and solids. It remains to establish whether these spatial oscillations affect the thermal self-diffusion of the grains as well. Because the wall affects the velocity distribution function in its neighborhood [45], it may change the form of the self-diffusivity in Eq. (5). Unfortunately, to our knowledge, no kinetic theory has yet captured this effect, which may be responsible for deviations of the model from numerical simulations at low $K_{s} / K_{g}$ in Fig. 5 .

Finally, we showed that heat transfer in the exchange limit can be further augmented by granular agitation, if the latter is intense enough to induce velocity fluctuations in the gas. We proposed a model for such augmentation that is based on the measurements of Verberg and Koch [43] in Lattice-Boltzmann numerical simulations of dense suspensions. In this model, we replaced the molecular conductivity of the gas by a higher conductivity arising from particleinduced gas velocity fluctuations. Part II will test the merit of this approach using experiments with grains vibrated in a box in air, for which such particle-induced augmentation of conductivity must be taken into account. Part III will focus on the diffusion limit, which is relevant to colloidal suspensions or to experiments in microgravity.

\section{Acknowledgements}

The authors are grateful to Haitao $\mathrm{Xu}$, Donald Koch, Rolf Verberg, Kenneth Torrance, Melany Hunt, Stephen Pope and James Jenkins for fruitful discussions. This work was sponsored by NASA grants NAG3-2705 and NCC3-797.

\section{REFERENCES}

1. L. R. Glicksman. Heat transfer in circulating fluidized beds, chapter in Circulating Fluidized Beds, J. Grace, T. Knowlton \& A. Avidan, eds., Blackie Academic \& Professional, London (1997), p. 261-311.

2. O. Molerus, K. Wirth. Heat transfer in fluidized beds. Chapman \& Hall, London, UK (1997).

3. G. Jepson, A. Poll, W. Smith. Heat transfer from gas to wall in a gas/solids transport line. Chemical Engineering Research and Design 41a (1963) 207-211.

4. J. S. Patton, R. H. Sabersky, C. E. Brennen. Convective heat transfer to rapidly flowing granular materials. Int. J. Heat Mass Transfer 29 (1986) 1263-1269.

5. V. V. R. Natarajan, M. L. Hunt. Heat transfer in vertical granular flows. Exp. Heat Transfer 10 (1997) 89-107.

6. M. Kaviany. Effect of a moving particle on wall heat transfer in a channel flow. Numerical Heat Transfer, Part A: Applications 13 (1988) 111-124.

7. A. S. Sangani, G. Mo, H. -K. Tsao, D. L. Koch. Simple shear flows of dense gassolid suspensions at finite Stokes numbers. $J$. Fluid Mech. 313 (1996) 309-341.

8. D. L. Koch, A. S. Sangani. Particle pressure and marginal stability limits for a homogeneous monodisperse gas fluidized bed: kinetic theory and numerical simulations. J. Fluid Mech. 400 (1999) 229-263.

9. H. Xu, M. Louge, A. Reeves. Solutions of the kinetic theory for bounded collisional granular flows. Continuum Mechanics and Thermodynamics 15 (2003) 321-349.

10. J. L. Sinclair, R. Jackson. Gas-particle flow in a vertical pipe with particle-particle interactions. AIChE J. 39 (1989) 1473-1486.

11. M. Y. Louge, E. Mastorakos, J. T. Jenkins. The role of particle collisions in pneumatic transport. J. Fluid Mech. 231 (1991) 345359.

12. S. Dasgupta, R. Jackson, S. Sundaresan. Turbulent gas-particle flow in vertical risers. AIChE J. 40 (1994) 215-228. 
13. E. J. Bolio, J. A. Yasuna, J. L. Sinclair. Dilute, turbulent gas-solid flow in risers with particle-particle interactions. AIChE J. 41 (1995) 1375-1388.

14. S. Sundaram, L. R. Collins. Collision statistics in an isotropic particle-laden turbulent suspension. Part 1. Direct numerical simulations. J. Fluid Mech. 335 (1997) 75-109.

15. J. T. Jenkins, D. M. Hanes. Collisional sheet flows of sediment driven by a turbulent fluid. J. Fluid Mech. 370 (1998) 29-52.

16. S. S. Hsiau, M. L. Hunt. Kinetic theory analysis of flow-induced particle diffusion and thermal conduction in granular material flows. ASME J. Heat Transfer 115 (1993) 541-548.

17. J. Sun, M. M. Chen. A theoretical analysis of heat transfer due to particle impact. Int. J. Heat Mass Transfer 31 (1988) 969-975.

18. M. Y. Louge, J. Mohd.-Yusof, J. T. Jenkins. Heat transfer in the pneumatic transport of massive particles. Int. J. Heat Mass Transfer 36 (1993) 265-275.

19. C. S. Campbell. Self-diffusion in granular shear flows. J. Fluid Mech. 348 (1997) 85101.

20. M. L. Hunt. Discrete element simulations for granular material flow: effective thermal conductivity and self-diffusivity. Int. J. Heat Mass Transfer 40 (1997) 3059-3068.

21. V. V. R. Natarajan, M. L. Hunt. Kinetic theory analysis of heat transfer in granular flows. Int. J. Heat Mass Transfer 41 (1998) 19291944.

22. J. C. Maxwell. Electricity and Magnetism, Clarendon Press, Oxford, UK (1873).

23. R. E. Meredith, C. W. Tobias. Resistance to potential flow through a cubical array of spheres. J. Appl. Phys. 31 (1960) 1270-1273.

24. R. T. Bonnecaze, J. F. Brady. The effective conductivity of random suspensions of spherical particles. Proc. Roy. Soc. Lond. A 432 (1991) 445-465.

25. N. F. Carnahan, K. E. Starling. Equation of state for non-attracting rigid spheres. $J$. Chem. Phys. 51 (1969) 635-636.

26. S. Torquato. Nearest-neighbor statistics for packings of hard spheres and disks. Phys. Rev. E 51 (1995) 3170-3182.
27. W. G. Vincenti, C. K. Kruger. Introduction to Physical Gas Dynamics. Krieger, NY (1965).

28. F. Ben-Ammar, M. Kaviany and J.R. Barber. Heat transfer during impact. Int. J. Heat Mass Transfer 35 (1992) 1495-1506.

29. J. K. Percus, G. J. Yevick. Analysis of Classical Statistical Mechanics by Means of Collective Coordinates. Physical Review $\mathbf{1 1 0}$ (1958) 1-13.

30. W. R. Smith, D. Henderson. Analytical representation of the Percus- Yevick hard-sphere radial distribution function. Molec. Phys. 19 (1970) 411-415.

31. D. Henderson, F. F. Abraham, J. A. Barker. The Onrstein-Zernike equation for a fluid in contact with a surface. Molec. Phys. 31 (1976) 1291-1293. Molec. Phys. 100 (2002) 129-132.

32. M. A. Hopkins, M.Y. Louge. Inelastic microstructure in rapid granular flows of smooth disks. Phys. Fluids A 3 (1991) 47-57.

33. F. P. Incropera, D. P. Dewitt. Fundamentals of Heat and Mass Transfer. John Wiley, 5th edition, NY (2002).

34. S. Shih. Numerical Heat Transfer. Hemisphere, Washington, DC (1984).

35. C. T. Crowe, T. R. Troutt, J. N. Chung. Numerical models for two-phase turbulent flows. Annu. Rev. Fluid Mech. 28 (1996) 11-43.

36. S. Sundaram, L. R. Collins. A numerical study of the modulation of isotropic turbulence by suspended particles. J. Fluid Mech. 379 (1999) 105-143.

37. M. Boivin, O. Simonin, K. D. Squires. On the prediction of gas-solid flows with two-way coupling using large eddy simulations. Phys. Fluids 12 (2000) 2080-2090.

38. O.A. Druzhinin. The influence of particle inertia on the two-way coupling and modification of isotropic turbulence by microparticles. Phys. Fluids 13 (2001) 3738-3755.

39. V. S. L'vov, G. Ooms, A. Pomyalov. Effect of particle inertia on turbulence in a suspension. Phys. Rev. E 67 (2003) 046314.

40. W. Hwang, J. K. Eaton. Homogeneous and isotropic turbulence modulations by small heavy $($ St $\sim 50)$ particles. J. Fluid Mech. 
564 (2006) 361-393.

41. G. Ooms, P. Poesio. Effect of particle inertia and gravity on the turbulence in a suspension. Phys. Fluids 17 (2005) 125101.

42. S. Whitaker. Forced convection heat transfer correlations for flow in pipes, past flat plates, single cylinders, single spheres, and for flow in packed beds and tube bundles. AIChE J. 18 (1972) 361-371.

43. R. Verberg, D. L. Koch. Rheology of particle suspension with low to moderate fluid inertia at finite particle inertia. Phys. Fluids $\mathbf{1 8}$ (2006) 083303.

44. J. T. Jenkins, M. W. Richman. Grad's 13moment system for a dense gas of inelastic spheres. Arch. Rat. Mech. Anal. 87 (1985) 355-377.

45. M. Y. Louge. Computer simulations of rapid granular flows of spheres interacting with a flat, frictional boundary. Phys. Fluids 6 (1994) 2253-2269. 